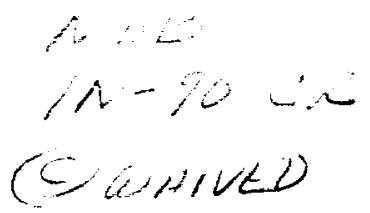

\title{
DUST GRAINS AND THE LUMINOSITY OF CIRCUMNUCLEAR WATER MASERS IN ACTIVE GALAXIES
}

\author{
Alan J. Collison and William D. Watson \\ Department of Physics, University of Illinois, 1110 West Green Street, Urbana, IL 61801

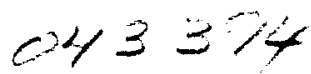 \\ Received 1995 June 12; accepted 1995 August 7
}

\begin{abstract}
In previous calculations for the luminosities of $22 \mathrm{GHz}$ water masers, the pumping is reduced and ultimately quenched with increasing depth into the gas because of trapping of the infrared $(\approx 30-150 \mu \mathrm{m})$, spectral line radiation of the water molecule. When the absorption (and reemission) of infrared radiation by dust grains is included, we demonstrate that the pumping is no longer quenched but remains constant with increasing optical depth. A temperature difference between the grains and the gas is required. Such conditions are expected to occur, for example, in the circumnuclear masing environments created by $\mathrm{X}$-rays in active galaxies. Here, the calculated $22 \mathrm{GHz}$ maser luminosities are increased by more than an order of magnitude. Application to the well-studied, circumnuclear masing disk in the galaxy NGC 4258 yields a maser luminosity near that inferred from observations if the observed $\mathrm{X}$-ray flux is assumed to be incident onto only the inner surface of the disk.

Subject headings: circumstellar matter — galaxies: active - masers
\end{abstract}

\section{INTRODUCTION}

Observations are accumulating to indicate that $22 \mathrm{GHz}$ water masers frequently occur in the gas near the nuclei of active galaxies (e.g., Braatz, Wilson, \& Henkel 1994). These masers have been particularly well studied in NGC 4258 (M106) since their detection (Claussen, Heiligman, \& Lo 1984) and provide a detailed description of a thin, circumnuclear disk in Keplerian motion at a radius of $0.1 \mathrm{pc}$ about a central mass of $4 \times 10^{7} M_{i ;}$ (Watson \& Wallin 1994; Miyoshi et al. 1995).

In contrast to water masers in our own galaxy (e.g., Watson 1994), the small deviations from Keplerian for the velocities of the individual components of the masing gas in NGC 4258 ( $\leq 10 \mathrm{~km} \mathrm{~s}^{1}$ ) suggest that shocks are not the main cause for circumnuclear masers. Calculations have also demonstrated that the observed flux of $\mathrm{X}$-rays can create regions of warm molecular gas at high densities in the circumnuclear environments of active galaxies, as is required for water masers (Krolik \& Lepp 1989). Neufeld, Maloney, \& Conger (1994; hereafter NMC) have shown in detail that the necessary conditions for masing of the $22 \mathrm{GHz}$ transition can be achieved and have calculated maser luminosities. The observation that most of the maser luminosity comes from the inner edge of the disk in NGC 4258 is also consistent with the idea that X-rays directly from a compact, nuclear source create the masing environment. Regardless of how the environment is created, inelastic collisions with $\mathrm{H}_{2}$ molecules and radiative cascades involving the rotational energy levels of the ground vibrational state (de Jong 1973) lead to the inverted population for the maser. The optical depths of the infrared transitions involved in the cascades are large because of reabsorption by other $\mathrm{H}_{2} \mathrm{O}$ molecules. With increasing optical depth, trapping of this radiation reduces and ultimately quenches the inversion. In the circumnuclear environments created by X-rays, this trapping significantly reduces the maser luminosities in the available calculations (see Fig. 2 of NMC). We reason in $\$ 3$ that previously calculated maser luminosities seem to be insufficient to create the observed luminosities, at least for NGC 4258 , where the refined data make possible a close comparison.
In this Letter we demonstrate how dust grains absorb the infrared radiation involved in the pumping cascade and prevent the quenching of the inversion described above. Absorption of the infrared radiation by dust in the pumping of $\mathrm{H}_{2} \mathrm{O}$ masers has previously been examined in the context of galactic masers (Deguchi 1981). For galactic water masers it has not seemed to be important because of the lower column densities for the regions of warm, masing gas. We focus on the circumnuclear disk of NGC 4258 because the detailed observational description of these masers provides the opportunity for a close comparison.

\section{CALCULATIONS}

To illustrate the influence of the grains, we present a direct comparison with the previous calculations. We thus use methods similar to those of NMC to calculate the emissivity $\Phi_{\text {s:t }}$ (photons $\mathrm{s}^{-1} \mathrm{~cm}^{-3}$ ) of a saturated maser as a function of depth into a thick slab of gas that is infinite in the directions parallel to the planar interface. Escape of the infrared radiation involved in determining the populations of the masing states is only allowed to occur through the interface and is treated using the "escape probability" approximation, in which there is no velocity gradient. To perform these computations, we have modified the code described elsewhere (Anderson \& Watson 1990) to incorporate the emission and absorption of infrared radiation by dust grains, to utilize static (rather than large velocity gradient [LVG]) escape probabilities, and to utilize more recent cross sections (Green, Maluendes, \& McLean 1993). By straightforward analysis, we find that the influence of the dust grains can be incorporated by modifying the usual rate equations (e.g., Anderson \& Watson 1993, eq. [11]) for the populations in the escape probability approximation to read

$$
\begin{aligned}
\sum_{j \neq i}\left(g_{j} C_{j i} n_{j}-\right. & \left.g_{i} C_{i j} n_{i}\right)+\sum_{j>i} g_{j} A_{j i}\left[n_{j}\left(\beta_{j i}+\beta_{j i}^{c}\right)+\left(n_{j}-n_{i}\right) S_{j i}^{c} \beta_{j i}^{c}\right] \\
& -\sum_{j<i} g_{i} A_{i j}\left[n_{i}\left(\beta_{i j}+\beta_{i j}\right)+\left(n_{i}-n_{j}\right) S_{i j}^{c} \beta_{i j}^{c}\right]=0
\end{aligned}
$$

in which $n_{i}$ and $n_{j}$ refer to the populations (per magnetic substate) of states $i$ and $j$ of $\mathrm{H}_{2} \mathrm{O}$ in the gas, $g_{i}$ and $g_{j}$ to the 
statistical weights, $A_{j i}$ to the Einstein $A$-values, and $C_{i j}$ to the rates of excitations (or de-excitations) due to collisions with molecular hydrogen (see also Hummer \& Rybicki 1985; Deguchi 1981). The summations are over states $j$. The terms that involve the escape probabilities $\beta_{i j}$ (and $\beta_{j i}$ ) in equation (1) describe the emission and absorption in the absence of dust grains. The terms that involve $\beta_{i j}^{c}$ (and $\beta_{j i}^{c}$ ) are modifications caused by the presence of grains. Note that $\beta_{i j}=\beta_{j i}, \beta_{i j}^{c}=\beta_{j i}^{c}$, and $S_{i j}^{c}=S_{j i}^{c}$. When the grains are much cooler than the gas, the source function due to grains, $S_{i j}^{c}=\left[\exp \left(h \nu_{i j} / k T_{d}\right)-1\right]^{-1}$ goes to zero, and the terms in equation (1) that involve $S_{i j}^{c}$ (and $\left.S_{j i}^{c}\right)$ disappear.

For convenience and consistency with previous work, we utilize the analytic approximations of Hollenbach \& McKee (1979; their eqs. [5.10] and [5.21]; note, however, that the reemission by dust was not treated there) for $\beta_{i j}$ and $\beta_{i j}^{c}$ :

$$
\begin{gathered}
\beta_{i j}=\left\{1+\tau_{i j}\left[2 \pi \ln \left(2.13+\tau_{i j}^{2}\right)\right]^{1 / 2}\right\}^{-1}, \\
\beta_{i j}^{c}=\delta_{i j}+\left[\frac{\left(1-\delta_{i j}\right) e^{-\tau_{d}}}{1+\left(1-\delta_{i j}\right) \tau_{d}^{2}}-1\right] \beta_{i j},
\end{gathered}
$$

where

$$
\delta_{i j}=\frac{2 \alpha_{i j}}{1+2 \alpha_{i j}}\left[\ln \left(2.72+\cdots \frac{1}{\pi^{1 / 2} \alpha_{i j}}\right)\right]^{1 / 2},
$$

in which $\tau_{i j}$ is the optical depth to the surface of the slab at the center of the $i j$ spectral line of $\mathrm{H}_{2} \mathrm{O}, \tau_{d}$ is the optical thickness to the edge of the slab due to dust at the wavelength of the $i j$ line, and $\alpha_{i j}=\tau_{d} / \tau_{i j}$. For $\tau_{d}$ we use an approximation based on Laor \& Draine (1993; see their Fig. 6) as a benchmark: $\tau_{d}=\tau_{0} N(\mathrm{H}) / 10^{23} \mathrm{~cm}^{-2}, \lambda<50 \mu \mathrm{m}$ and $\tau_{d}=\tau_{0}(50 \mu \mathrm{m} /$ $\lambda)^{2} N(\mathrm{H}) / 10^{23} \mathrm{~cm}^{-2}, \lambda>50 \mu \mathrm{m}$, in which $N(\mathrm{H})$ is the column density of hydrogen nuclei to the edge of the slab and $\tau_{0}=1$. Calculations are also performed with different $\tau_{0}$ and wavelength dependences to verify that modest uncertainty in the assumed properties of the grains leads to only modest changes in our results. There is, of course, uncertainty in $\tau_{d}$. To obtain $\Phi_{\text {sat }}$, the radiative terms in the rate equations (1) that represent the $22 \mathrm{GHz}$ maser transition are replaced by $-R\left(n_{u}-n_{l}\right)$ in the equation for the upper state $(i=u)$ and by $R\left(n_{u}-n_{l}\right)$ in the equation for the lower state $(i=l)$. Equations (1) are then solved numerically as a function of $R$ to obtain the limiting constant value $\Phi_{\text {sat }}=R\left(n_{u}-n_{l}\right)$ as $R \rightarrow \infty$. In previous investigations, $\Phi_{\mathrm{sat}}$ has been obtained by first computing $\left(n_{u}-n_{i}\right)$ from the rate equations in the unsaturated limit $(R=0)$ and then multiplying this quantity by an effective loss rate. Potential ambiguities arise (at least to us) in determining an effective loss rate. However, with the loss rate as specified in Anderson \& Watson (1993), we find that the two methods yield similar $\Phi_{\text {sat }}$ values for the regions of most interest here.

To perform the calculations, the gas density, kinetic temperature, and $\mathrm{H}_{2} \mathrm{O}$ fractional abundance within the slab of gas must be specified. Fortunately, these quantities have already been computed by NMC (see their Fig. 2) for an incident $\mathrm{X}$-ray flux that is essentially identical with that inferred for the X-ray luminosity of NGC $4258\left(\approx 10^{41}\right.$ ergs $\mathrm{s}^{-1}$ above $1 \mathrm{keV}$; Makishima et al. 1994) incident onto a gas with a pressure of $10^{11} \mathrm{~K} \mathrm{~cm}^{-3}$ at the distance of the inner radius of the masing disk $(0.1 \mathrm{pc})$. In Figure 1 , we present the results of our calculations performed in the manner described above. For comparison, we also show the results of similar calculations from Figure 2 of NMC. The general agreement between our

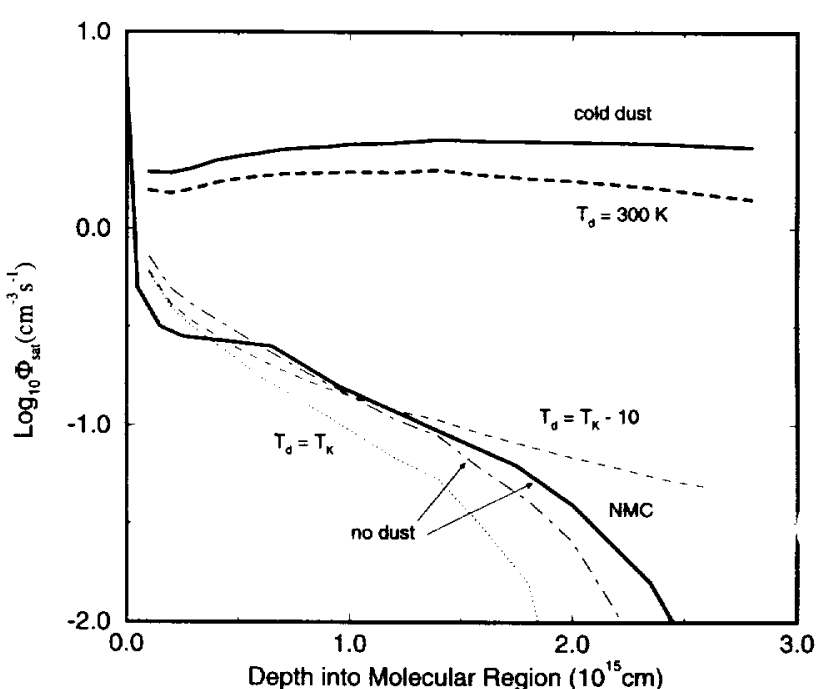

FIci. 1.- The log of the maser photon emissivity vs. distance into a slab of gas that is irradiated by an X-ray flux similar to that expected for the circumnuclear masing gas in the galaxy NGC 4258 at a gas (pressure $/ k$ ) $=10^{11}$ $\mathrm{K} \mathrm{cm}{ }^{3}$. The expected enhancement due to dust is best seen by comparing the solid line (NMC), which was obtained previously in a similar calculation but without dust, to the solid line labeled "cold dust." Calculations are also presented for various temperatures $T_{d}$ for the dust $\left(T_{k}=\right.$ gas temperature). Our curve (dot-dashed line) best reproduces the calculations of NMC.

calculations for $\Phi_{\mathrm{sat}}$ with no dust and the analogous calculations by NMC is evidence of the general consistency of the calculational methods (but see below).

The influence of dust on $\Phi_{\text {sar }}$ is illustrated by our other calculations presented in Figure 1 . It is seen most clearly by comparing the two solid lines which, when integrated to an appropriate depth to obtain the maser luminosity, differ by a factor of about 20 (see $\$ 3$ ). Calculations are also presented in which the dust temperature is $300 \mathrm{~K}$ and in which it is equal to the the gas kinetic temperature $T_{K}$ and differs from $T_{K}$ by $10 \mathrm{~K}$. Modest changes (up to a factor of 3 or so) in the velocity dispersion $\Delta v$ or the dust parameter $\tau_{\| 1}$ will result in comparable fractional changes in $\Phi_{\text {sat }}$. In $\S 3$ we reason that the grain temperature should be about $200 \mathrm{~K}$ in the masing gas for NGC 4258 , and thus the presence of dust can cause a major increase in the total maser luminosity. The result in Figure 1 that $\Phi_{\text {sat }}$ is essentially constant (except near the boundary) when dust is present reflects the fact that absorption is more important than escape for infrared spectral line radiation. Once the column density of $\mathrm{H}_{2} \mathrm{O}$ to the boundary is large enough to cause sufficient scatterings that the accumulated path length of a photon becomes equal to the mean free path for absorption by dust (which occurs when $\tau_{d} \approx 0.1$ ), further increase in the column density of $\mathrm{H}_{2} \mathrm{O}$ (and hence the depth into the slab) is unimportant. After this number of scatterings, the photon is destroyed through absorption by dust grains. The result that $\Phi_{\text {sal }}$ becomes constant can also be seen in equations (1), (3), and (4). With increasing depth into the slab, $\boldsymbol{\beta}_{i j}^{c}$ dominates $\boldsymbol{\beta}_{i j}$ and becomes independent of depth,

$$
\beta_{i j}^{c} \rightarrow\left(2 \tau_{d} / \tau_{i j}\right)\left\{\ln \left[2.72+\left(\tau_{i j} / \pi^{1 / 2} \tau_{d}\right)\right]\right\}^{1 / 2},
$$

since the ratio $\tau_{d} / \tau_{i j}$ is independent of depth. Equation (1) and its solutions must then also become independent of depth into the slab, as seen in Figure 1. The presence of dust thus results in a lower limit to the escape probability. In detail, our calculations for $\Phi_{\text {sat }}$ in Figure 1 are somewhat different from 
those of NMC. This is partly compensated by our use of $\Delta v=2 \mathrm{~km} \mathrm{~s}^{-1}$ versus their $\Delta v=1 \mathrm{~km} \mathrm{~s}^{-1}$. After the original version of this Letter was submitted, D. Neufeld and P. Maloney kindly made available the exact variation with depth of the physical conditions used for calculating $\Phi_{\text {sar }}$. When these are used, we have verified that the effect of dust is changed negligibly from that indicated in Figure 1.

\section{DISCUSSION}

Most of the radiation from a compact nuclear source (optical, ultraviolet, soft $\mathrm{X}$-rays) will be absorbed near the inner interface when the slab of gas has an optical depth greater than a few at $50 \mu \mathrm{m}$, as is relevant here. This energy will be processed into infrared radiation that will be absorbed (and reemitted) to maintain the temperatures of the dust grains in the slab. The benchmark temperature for the dust grains is then the effective blackbody temperature of the bolometric flux $F_{b}$ of radiant energy incident onto the inner surface $\left(=\left[F_{b} / 4 \sigma\right]^{1 / 4}\right)$. Calculations seem to indicate that grains in thick slabs have essentially this temperature beyond an optical depth of a few tenths (see Fig. 14 of Laor \& Draine 1993). Making the assumption that the ratio of the bolometric luminosity to the $\mathrm{X}$-ray luminosity is about 10 , as is typical for active nuclei, then gives a dust temperature $T_{d} \approx 200 \mathrm{~K}$ within the masing gas at a distance of $0.13 \mathrm{pc}$ from the compact source in NGC 4258. In contrast, when this X-ray flux is incident onto the interface of the slab of gas used in Figure 1, it creates the gas kinetic temperatures of $\approx 400-600 \mathrm{~K}$ that are used in the calculations for Figure 1. Although the grains and the gas exchange energy through collisions and through the emission and absorption of infrared radiation, the additional energy input to the gas due to photoionization by the $\mathrm{X}$-rays causes the calculated temperature difference. Based on the above information, this difference in temperature between the grains and the gas is large enough that the $\Phi_{\text {sat }}$ values calculated in the "cold dust" limit are adequate approximations.

To obtain the true (i.e., integrated over angles of emission) maser luminosity to compare with observations, $\Phi_{\text {sat }}$ must be integrated over the masing volume. For a ring of thickness $h$ with an inner radius $R$, the maser luminosity is

$$
L_{m}=2 \pi \mathrm{h} \nu R h \int \Phi_{\text {sat }} d R
$$

where the radial extent of the masing gas is recognized to be much less than $R$. Beyond the depth at which the optical depth for Thomson scattering is about $1\left[N(\mathrm{H})=1.5 \times 10^{24} \mathrm{H}\right.$ nuclei $\mathrm{cm}^{-2}$, the X-ray heating is significantly decreased. We thus terminate the integral in equation (5) at this depth, which corresponds to $4 \times 10^{15} \mathrm{~cm}$ in Figure 1. For the "cold dust" curve in Figure $1, \mathrm{~h} v \int \Phi_{\text {sat }} d R$ is thus $3800 L_{0} \mathrm{pc}^{-2}$. Then, from the published observations of the disk in NGC $4258[R=0.13$ pc and $(h / R)<0.02$ ], $L_{m} \leq 8 L_{\%}$, in agreement with the observed luminosity of $7 L_{\text {(.) }}$ based on the inferred beaming angle (Miyoshi et al. 1995, Table 1). Our value for $\mathrm{h} \nu \int \Phi_{\text {sat }} d R$, and hence for $L_{m}$ as well, exceeds that of NMC $(=180 L$ $\mathrm{pc}^{-2}$ ), which was computed for similar conditions, by a factor of 20. From a similar analysis, NMC concluded that their lower value of $\mathrm{h} \nu \int \Phi_{\text {sat }} d R$ is compatible with the observations. The discrepency arises in part because the beaming angle adopted by NMC $(\Omega=2 \pi h / R)$ is smaller than that inferred from the observations of NGC 4258. In the interpretation of Miyoshi et al. (1995), the beaming angle is determined by the size of a nuclear continuum source, which is being amplified by the central masers. This source is larger than the thickness of the disk. Since the continuum source has not yet been detected, potential uncertainty remains about this interpretation for the beaming angle. However, $\Omega \approx 2 \pi h / R$ is based on the premise that the gas that creates most of the observed maser flux (the nearly radial central emission) is distributed roughly uniformly over a radial extent of the disk that is comparable to $R$. In contrast, Miyoshi et al. (1995) seem to find that the radial extent of this masing gas is much smaller than $R$.

Because of the narrowness of the observed spectral lines of the circumnuclear water masers (as small as a few kilometers per second), the calculations to date have only utilized similarly small velocity dispersions in calculating escape probabilities. If velocity fields with larger dispersion are present that are not reflected in the spectral line profiles, the calculated maser luminosity will be increased. Adopting a larger velocity dispersion in directions orthogonal to the line of sight is evidently one such possibility. The thinness of the observed disk and the apparent need for hydrostatic equilibrium does, however, limit such additional velocity dispersion. The foregoing analysis is based on the premise (as was NMC) that a significant $X$-ray flux is incident only on the inner surface of the disk of thickness $h$. If X-rays incident on the upper and lower surfaces of the disk due to scattering by the halo or to incidence at grazing angles directly from the central source are important, it is evident that the masing volume will be increased over that included in equation (5) and the maser luminosity will be appropriately increased. However, quantitative assessments of these possibilities have not yet been presented. At gas pressures greater than the $10^{11} \mathrm{~K} \mathrm{~cm}{ }^{3}$ considered in Figure 1, the extent (in column density of hydrogen) of the molecular gas created by X-rays that is warm enough for masing is reduced. According to NMC, at (pressure $(k)=10^{12} \mathrm{~K} \mathrm{~cm}^{-3}$ the gas is cool enough that masing is unimportant at depths into the slab for which absorption by dust is important in the pumping. Finally, the absorption and emission by dust at the wavelengths of the spectral lines of the gas can influence the cooling of the gas, as well as the pumping of the maser. However, examination of the cooling rates (Fig. 6 of Neufeld \& Kaufman 1993) suggests that this will be of minor importance for the conditions on which Figure 1 is based.

This research has been supported in part by NSF grant AST94-01348 and NASA grant NAGW-1104. We are especially grateful to P. R. Maloney and D. A. Neufeld for information about the physical conditions in these X-radiated molecular gases and about the calculation of $\boldsymbol{D}_{\mathrm{v}, t}$ in NMC.
Anderson, N., \& Watson, W. D. 1990, ApJ, 348, L69 1993, ApJ, 407, 620

Braatz, J. A., Wilson, A. S., \& Henkel, C. 1994, ApJ, 437, L99

Claussen, M. J., Heiligman, G. M., \& Lo, K. Y. 1984, Nature, 310, 298

\section{REFERENCES}

Deguchi, S. 1981, ApJ, 249, 145 de Jong, T. 1973, A\&A, 26, 297

Green, S., Maluendes, S., \& McLean, A. D. 1993, ApJS, 85, 181

Hollenbach, D., \& McKee, C. F. 1979, ApJS, 41, 555 
Hummer, D. G., \& Rybicki, G. B. 1985, ApJ, 293, 258

Krolik, J. H., \& Lepp, S. 1989, ApJ, 347, 179

Laor, A., \& Draine, B. T. 1993, ApJ, 402, 441

Makishima, K., et al. 1994, PASJ, 46, L77

Miyoshi, M., Moran, J., Herrnstein, J., Greenhill, L., Nakai, N., Diamond, P., \& Inoue, M. 1995, Nature, 373, 127
Neufeld, D. A., \& Kaufman, M. J. 1993, ApJ, 418, 263 Neufeld, D. A., Maloney, P. R., \& Conger, S. 1994, ApJ, 436, L127 (NMC) Watson, W. D. 1994, in Lecture Notes in Physics, Vol. 439, The Structure and Content of Molecular Clouds, ed. T. L. Wilson \& K. J. Johnston (Berlin: Springer), 109

Watson, W. D., \& Wallin, B. K. 1994, ApJ, 432, L35 